\title{
Comments on the Regular and Irregular IsoRepresentations of the Lie-Santilli IsoAlgebras
}

\author{
Richard Anderson \\ The R. M. Santilli Foundation, Palm Harbor, Florida, U.S.A.
}

Email address:

board@santilli-foundation.org

To cite this article:

Richard Anderson. Comments on the Regular and Irregular IsoRepresentations of the Lie-Santilli IsoAlgebras. American Journal of Modern Physics. Special Issue: Issue I: Foundations of Hadronic Mathematics. Vol. 4, No. 5-1, 2015, pp. 76-82.

doi: 10.11648/j.ajmp.s.2015040501.19

\begin{abstract}
As it is well known, 20th century applied mathematics with related physical and chemical theories, are solely applicable to point-like particles moving in vacuum under Hamiltonian interactions (exterior dynamical problems). In this note, we study the covering of 20th century mathematics discovered by R. M. Santilli, today known as Santilli isomathematics, representing particles as being extended, non-spherical and deformable while moving within a physical medium under Hamiltonian and non-Hamiltonian interactions (interior dynamical problems). In particular, we focus the attention on a central part of isomathematics given by the isorepresentations of the Lie-Santilli isoalgebras that have been classified into regular (irregular) isorepresentations depending on whether the structure quantities of the isocommutation rules are constants (functions of local variables). The importance of the study of the isorepresentation theory for a number of physical and chemical applications is pointed out.
\end{abstract}

Keywords: Lie Theory, Lie-Santilli Isotheory, Isorepresentations

\section{Introduction}

As it is well known, 20th century applied mathematics at large, and the Lie theory in particular, can only represent point-like particles moving in vacuum (exterior dynamical problems), resulting in a body of methods that have proved to be effective whenever particles can be effectively abstracted as being point-like, such as for the structure of atoms, and crystals, particles moving in accelerators, and many other systems.

An important feature of exterior problems is that, being dimensionless, point-like particles can only experience action-at-a-distance, potential and, therefore, Hamiltonian interactions, which Hamiltonian character is a central condition for the very applicability of Lie's theory.

It is equally well known that point-like abstractions of particles are excessive for extended, non-spherical and deformable particles moving within a physical medium (interior dynamical problems), as it is the case for the structure of hadrons, nuclei and stars since their constituents are in a state of mutual penetration of their wavepackets and/or charge distribution.

An important feature of the finite size of particles in interior conditions is that they experience conventional action-a-distance, Hamiltonian interactions, as well as additional contact, non-potential and, therefore, non-Hamiltonian interactions, with the consequential inapplicability of 20th century applied mathematics at large, and of Lie's theory in particular.

In a series of pioneering works [1-11], R. M. Santilli has constructed a new mathematics, today known as Santilli IsoMathematics, for the representation of extended, non-spherical and deformable particles under Hamiltonian as well as non-Hamiltonian interactions, which new mathematics has seen contributions by numerous important mathematicians (see, e.g. Rfs., [12-21]).

In this note, the author would like to bring to the attention of the mathematical community the need for further studies on the central branch of isomathematics, namely, the Lie-Santilli IsoTheory [1], since the latter provides the only known time invariant methods for the lifting of the various applications of the conventional Lie theory from exterior to interior conditions.

In particular, we focus the attention on the 
IsoRepresentations of the Lie-Santilli IsoAlgebras which have been classified into regular and irregular, depending on whether the structure quantities of the isocommutation rules are constant or functions of the local variables.

Besides Santilli's works, no study on the isorepresentation theory of the Lie-Santilli isoalgebras is on scientific record to our best knowledge, with consequential limitations on important applications, such as the search for much needed, new nuclear energies without the release of harmful radiations and other equally important applications outlined in Section 5.

It should be indicated that Santilli's pioneering works signal the historical transition from the notion of massive point, introduced by Newton, and adopted by Galileo and Einstein, to a new generation of physical and chemical theories representing particles as they are in the physical or chemical reality. This historical advance has so many implications for all of quantitative sciences that it has been referred to as characterizing New Sciences for a New Era in the title of Ref. [21].

\section{The Lie-Santilli IsoTheory}

Let $\mathrm{L}$ be an $\mathrm{N}$-dimensional Lie algebra on a Hilbert space $H$ over a field $F(n, \times, 1)$ with elements $n$ given by real, complex and quaternionic numbers, associative product hereon denoted $\mathrm{nm}=\mathrm{n} \times \mathrm{m} \in \mathrm{F}$, and multiplicative unit 1 .

Let the generators of $\mathrm{L}$ be given by Hermitean operators $\mathrm{X}_{\mathrm{k}}, \mathrm{k}=1,2, \ldots, \mathrm{N}$, on $\mathrm{H}$ over $\mathrm{F}$. Let $\xi$ be the universal enveloping associative algebra characterized by the infinite-dimensional set of ordered monomials according to the Poincaré-Birkhoff-Witt Theorem.

Let the Lie algebra $\mathrm{L}$ be isomorphic to the anti-symmetric algebra attached to the enveloping algebra $\mathrm{L} \approx \xi^{-}$with ensuing Lie's theorems and commutation rules. Let $\mathrm{G}$ be the Lie transformation group characterized by $\mathrm{L}$.

In pioneering works done in 1978-1983 at the Department of Mathematics of Harvard University under DOE support, R. M. Santilli [1] proposed the axiom-preserving isotopies of 20th century applied mathematics at large, and of the Lie theory in particular, via the following isotropy of the associative product

$$
X_{i} \widehat{\times} X_{j}=X_{i} \times \widehat{T} \times X_{j}
$$

where $\hat{\mathrm{T}}$, called the isotopic element, is solely restricted to be positive-definite, but otherwise possesses an arbitrary dependence on local variables such as time $t$, coordinates $r$, velocities $\mathrm{v}$, density $\mu$, temperature $\tau$, index of refraction $\delta$, frequency $\omega$, wave functions $\psi$, etc.

The fundamental significance of Santilli's infinite class of isotopies (1) of the associative product is that they permit the representation of the actual extended, and deformable shape of the body considered under Hamiltonian interactions represented via the conventional Hamiltonian, and contact non-Hamiltonian interactions via realizations of the isotopic element of the type

$$
\widehat{T}=\operatorname{Diag} \cdot\left(\frac{1}{n_{1}^{2}}, \frac{1}{n_{2}^{2}}, \frac{1}{n_{3}^{2}}\right) \times e^{-\Gamma(t, r, \mathcal{v}, \mu, \tau, \delta, \omega, \psi, \ldots)}
$$

where $n_{k}^{2}=n_{k}^{2}(t, r, v, \mu, \tau, \delta, \omega, \psi, \ldots) ! k=1,2,3$, represents, in this case, the deformable semi-axes of the considered ellipsoid, and $\Gamma$ is a positive-definite function representing all interactions not representable with the Hamiltonian.

Following the above basic assumptions, Santilli passed in monographs [1] to the construction of the isotopies of the various branches of Lie's theory over a conventional field F, and illustrated its significance via the Birkhoffian generalization of Hamiltonian mechanics which achieves "direct universality" for the representation of all possible (regular) non-Hamiltonian Newtonian systems directly in the frame of the experimenter. The resulting new theory is today known as the Lie-Santilli IsoTheory.

Following the above seminal advances, Santilli discovered that the original formulation [1] of the isotopies does not predict the same numerical values under the same conditions at different times (hereon referred to as time invriance), because the time evolution is non-unitary on $\mathrm{H}$ over $\mathrm{F}$.

In summer 1993, while visiting the Joint Institute for Nuclear Research in Dubna, Russia, Santilli [2] discovered that the abstract axioms of a numeric field do not necessarily require that the basic unit be the number 1 , since the multiplicative unit can be an arbitrary, positive definite quantity $\hat{\mathrm{I}}$ irrespective of whether an element of the original field $\mathrm{F}$ or not, under the condition that it is the inverse of the isotopic element

$$
\hat{I}=1 / \widehat{T},
$$

and all possible associative products are lifted into form (1) under which $\hat{\mathrm{I}}$ is the correct left and right multiplicative unit for all elements of the set considered

$$
\hat{I} \widehat{\times} X=X \widehat{\times} \hat{I}=X \forall X \in L
$$

This lead to the discovery of new fields, today known as Santilli isofields $\widehat{F}(\widehat{n}, \widehat{\times}, \hat{I})$ with isoreal, isocomplex and isoquaternionic isonumbers $\widehat{n}=n \times \hat{I}, n \in F$ equipped with the isoproduct $\widehat{n} h a t \times \widehat{m}=n \times m \times \widehat{I} \in \widehat{F}$ [2].

Subsequently, Santilli discovered that, despite the reformulation over an isofield, the Lie-Santilli isotheory was still unable to achieve the crucial time invariance of the numerical prediction.

Following various trials and errors, while studying at the Institute for Basic research, Castle Prince Pignatelli in Italy, Santilli [3] discovered in 1995 that, contrary to a popular belief in mathematics and physics for centuries, the Newton-Leibnitz differential calculus depends indeed on the assumed basic multiplicative unit because, in the event such unit has a functional dependence on the differentiation variable, the conventional differential must be generalized into the isodifferential

$$
\hat{d} \hat{r}=\hat{T} \times d[r \times \hat{I}(r, \ldots)]=d r+r \times \hat{T} \times d \hat{I}(r, \ldots),
$$

with ensuing isoderivative 


$$
\frac{\widehat{\partial} \hat{f}(\hat{r})}{\widehat{\partial} \hat{r}}=\hat{I} \times \frac{\partial \hat{f}(\hat{r})}{\partial \hat{r}} .
$$

where, for consistency, $\widehat{f}$ is an isofunction with the structure $\widehat{f}=f \times \hat{I}$ and $\hat{r}$ is the isovariable with the structure $\hat{r}=r \times \hat{I}$ as an evident condition to have values in the isofield $\widehat{F}$.

The discovery of isofields and of the isodifferential calculus signed the achievement in memoir [3] of mathematical maturity in the formulations of the isotopies of 20th century applied mathematics at large, and of the Lie-Santilli isotheory in particular, which maturity stimulated seminal, advances in mathematics as well as in physics and chemistry, including novel industrial applications indicated later on.

Nowadays, the Lie-Santilli IsoTheory is referred to the infinite family of isotopies of Lie's theory as defined in memoir [3], namely, formulated on an iso-Hilbert space $\widehat{H}$ over an isofield $\widehat{F}$ with iso-Hermitean generators $X_{k}, k=1,2, \ldots, N$, with all possible products lifted into the isoassociative form (1) and multiplicative isounit (3), the elaboration beng done via the isofunctional analysis and the isodifferential calculus.

A rudimentary outline of the Lie-Santilli isotheory comprises the following main branches $[3,9]$ :

2.1) The universal enveloping isoassociative isoalgebra $\hat{\xi}$ with infinite-dimensional isobasis given by the ordered isomonomials of the Poincaré-Birkhoff-Witt-Santilli isotheorem

$$
\hat{I}, X_{k}, X_{i} \times X_{j}, i \leq j, \ldots .
$$

with related isoexponentiation

$$
\hat{e}^{X}=\hat{I}+\frac{X}{\hat{1} !}+\frac{X \widehat{\times} X}{\widehat{2} !}+\cdots=\left(e^{X \times \hat{T}}\right) \times \hat{I}=\hat{I} \times\left(e^{\widehat{T} \times X}\right)
$$

and other isofunctions;

2.2) The Lie-Santilli isoalgebras

$$
\hat{L} \approx \hat{\xi}^{-}
$$

with isocommutation rules

$$
\left[X_{i} \hat{,} X_{j}\right]=X_{i} \widehat{\times} X_{j}-X_{j} \widehat{\times} X_{i}=\hat{C}_{i j}^{k} \widehat{\times} X_{k}
$$

where $\hat{C}_{i j}^{k}=C_{i j}^{k} \times \hat{I}$ are the isostructure quantities of $\widehat{L}$ with values in $\widehat{F}$;

2.3) The Lie-Santilli isogroups $\widehat{G}$ with structure for the one dimensional case ()

$$
\begin{aligned}
& \hat{A}(\widehat{W})=\hat{e}^{\widehat{H} \widehat{\times} \widehat{W} \widehat{\times} \hat{\imath}} \widehat{\times} \hat{A}(\widehat{0}) \widehat{\times} \hat{e}^{(-\hat{\imath} \widehat{\times} \widehat{w} \widehat{\times} \widehat{H})}= \\
& =e^{H \times \hat{T} \times w \times i} \times A(0) \times e^{-i \times w \times] \widehat{T} \times H}
\end{aligned}
$$

where $\widehat{H}=H \times \hat{I}$ is an isomatrix, namely, a matrix whose elements are isoscalars. The remaining aspect of the Lie-Santilli isotheory can be then constructed via axiom preserving isotopies of the totality of the conventional formulations with no exception known to the author.

Following the achievement in memoir [3] of a consistent formulation of the isotopies, Santilli applied the isotopies of Lie's isotheory them to a number of physical and chemical problems that cannot be even formulated with conventional Lie theory due to the need to represent of extended bodies under non-Hamiltonian interactions (see applications [6.7.8] with corresponding independent verifications and industrial applications [12,13,14], monograph [9] for a general treatment of the Lie-Santilli isotheory, and monographs [10,11] for applications in physics and chemistry, respectively).

In the author's view, Santilli's most salient achievement has been, not only the transition from the massive points of Newton, Galileo and Einstein theories to extended bodies, but also their representation under the most general (but non-singular) known non-linear, non-local and non-Hamiltonian interactions in a way as invariant as Hamiltonian formulations are.

This historical result was achieved via the embedding of all non-Hamiltonian quantities in the generalized unit of the theory because, whether conventional or generalized, the unit is indeed the basic invariant of any theory.

\section{Classification of IsoRepresentations}

The isorepresentations of Lie-Santilli isoalgebras are classified into $[4,5,9]$ :

3.1) Regular isorepresentations occurring when the C 's of rules (5) are constant; and

3.2) Irregular isorepresentations occurring when the C's of rules (5) are functions of local variables.

We should recall that "structure functions" are impossible for Lie's theory, and they are solely possible for the covering Lie-Santilli isotheory, by therefore establishing the non-trivial character of Santilli isotopies.

\section{Regular IsoRepresentations}

Let us recall that a given Lie algebra admits an infinite family of isotopies because a point-like particle in vacuum admits an infinite number of generalizations to extended particles moving within physical media.

Let us also recall that the extended shape of a particle and its non-Hamiltonian interactions are represented by the basic isounit or, equivalently, by the isotopic element [2].

Therefore, the transition from the conventional representations of a Lie algebra to the isorepresentation of the covering Lie-Santilli isoalgebras represents extended particles moving within physical media under conventional Hamiltonian interactions, as well as the most general known non-linear, non-local and non-Hamiltonian interactions.

Consider a given Lie algebra $\mathrm{L}$ and one of its representations. Santilli $[4,5,9]$ has identified a simple method for the construction of the infinite family of regular isorepresentations of the Lie-Santilli covering $\hat{\mathrm{L}}$ of $\mathrm{L}$ based on non-unitary transformations of the original Lie formulation. The method consists in:

4.1) Identifying the extended character of the particle considered and its non-Hamiltonian interactions represented via Santilli's isounit. 
4.2) The identification of a non-unitary transform representing said isounit according to the rule

$$
U \times U^{\dagger}=\hat{I}
$$

where

$$
U \times U^{\dagger} \neq I,
$$

4.3) The application of the above nonunitary transform to the totality of the mathematics underlying the original representation of $\mathrm{L}$, thus including numbers, spaces, algebras, geometries, symmetries, etc, with no known exception.

The bove method is illustrated by the transformations:

$$
\begin{aligned}
& I \rightarrow \hat{I}=U \times I \times U^{\dagger}=1 / \widehat{T}, \\
& n \rightarrow \hat{n}=U \times n \times U^{\dagger}=n \times U \times U^{\dagger}=n \times \hat{I} \in \hat{F},, n \in F, \\
& e^{A} \rightarrow U \times e^{A} \times U^{\dagger}=\hat{I} \times e^{\hat{T} \times \hat{A}}=\left(e^{\hat{A} \times \hat{T}}\right) \times \hat{I}, \\
& A \times B \rightarrow U \times(A \times B) \times U^{\dagger}=\left(U \times A \times U^{\dagger}\right) \times \\
& \left(U \times U^{\dagger}\right)^{-1} \times\left(U \times B \times U^{\dagger}\right)=\hat{A} \widehat{\times} \hat{B}, \\
& {\left[X_{i}, X_{j}\right] \rightarrow U \times\left[X_{i} X_{j}\right] \times U^{\dagger}=\left[\hat{X}_{i}, \hat{X}_{j}\right]=} \\
& U \times\left(C_{i j}^{k} \times X_{k}\right) \times U^{\dagger}=\hat{C}_{i j}^{k} \widehat{\times} \hat{X}_{k}=C_{i j}^{k} \times \hat{X}_{k}, \\
& <\psi|\times| \psi>\rightarrow U \times<\psi|\times| \psi>\times U^{\dagger}=< \\
& \psi\left|\times U^{\dagger} \times\left(U \times U^{\dagger}\right)^{-1} \times U \times\right| \psi>\times\left(U \times U^{\dagger}\right)=< \\
& \widehat{\psi}|\widehat{\times}| \widehat{\psi}>\times \hat{I}, \\
& H \times \mid \psi>\rightarrow U \times(H \times \mid \psi>)= \\
& \left(U \times H \times U^{\dagger}\right) \times\left(U \times U^{\dagger}\right)^{-1} \times(U \times \mid \psi>)= \\
& \widehat{H} \widehat{\times} \mid \widehat{\psi}>, e t c .
\end{aligned}
$$

As an illustration, Santilli considered in Refs. [4,5] the two-dimensional irreducible representation of the $\mathrm{SU}(2)$ Lie algebra, which is given by the known Pauli matrices.

The regular isorepresentations of the Lie-Santilli isoalgebras $\widehat{S U}(2)$ can be constructed via the infinite family of non-unitary transformations with representative example

$$
\begin{gathered}
\hat{\sigma}_{k}=U \times \sigma_{k} \times U^{\dagger}, \\
U=\left(\begin{array}{cc}
i \times g_{1} & 0 \\
0 & i \times g_{2}
\end{array}\right), \\
U^{\dagger}=\left(\begin{array}{cc}
-i \times g_{1} & 0 \\
0 & -i \times g_{2}
\end{array}\right), \\
g_{1}^{2}=\frac{1}{g_{2}^{2}}=\lambda^{2},
\end{gathered}
$$

where conditions (15c) is necessary for the isounitarity of the algebra and the $\mathrm{g}$ 's are well behaved nowhere null functions. The application of transformations (14) yields the regular
Pauli-Santilli isomatrices [4,5,9]

$$
\begin{gathered}
\hat{\sigma}_{1}=\left(\begin{array}{cc}
0 & g_{1}^{2} \\
g_{2}^{2} & 0
\end{array}\right), \\
\hat{\sigma}_{2}=\left(\begin{array}{cc}
0 & -i \times g_{1}^{2} \\
i \times g_{2}^{2} & 0
\end{array}\right), \\
\hat{\sigma}_{3}=\left(\begin{array}{cc}
g_{1}^{2} & 0 \\
0 & g_{2}^{2}
\end{array}\right) .
\end{gathered}
$$

with isoalgebra isomorphic to the conventional $S U(2)$ algebra

$$
\left[\hat{\sigma}_{i}, \hat{\sigma}_{j}\right]=\hat{\sigma}_{i} \times \hat{T} \times \hat{\sigma}_{j}-\hat{\sigma}_{j} \times \hat{T} \times \hat{\sigma}_{i}=2 \times i \times \varepsilon_{i j k} \times \hat{\sigma}_{k},
$$

and consequential preservation of the conventional eigenvalues for spin $1 / 2$

$$
\begin{array}{r}
\hat{\sigma}^{\widehat{2}} \widehat{\times} \mid \hat{\psi}>=\left(\hat{\sigma}_{1} \times T \times \hat{\sigma}_{1}+\hat{\sigma}_{2} \times T \times \hat{\sigma}_{2}+\right. \\
\left.+\hat{\sigma}_{3} \times T \times \hat{\sigma}_{3}\right) \times T \times|\hat{\psi}>=3 \times| \hat{\psi}>, \\
\hat{\sigma}_{3} \widehat{\times}\left|\hat{\psi}>=\hat{\sigma}_{3} \times T \times\right| \hat{\psi}>= \pm 1 \times \mid \hat{\psi}>,
\end{array}
$$

Despite the apparent triviality, Santilli's isotopies of the $\mathrm{SU}(2)$-spin algebra are not trivial because they introduce a new degree of freedom in the conventional spin $1 / 2$ given by the non-singular, but unrestricted parameter (or function) $\lambda^{2}$ of Eqs. (15c).

In turn, this new degree of freedom has permitted a number of novel applications, such as $[4,5,9]$ : the reconstruction of the exact isospin symmetry in nuclear physics which was believed to be broken by weak interactions; the achievement of a concrete and explicit realization of hidden variables in quantum mechanics via the degrees of freedom $\lambda^{2}$; and rather seminal implications for local realism (see Ref. [5] for brevity).

\section{Irregular IsoRepresentations}

Santilli has additionally constructed in Refs. [4,5] the following example of irregular isorepresentation of the ŜU(2) spin algebra

$$
\begin{gathered}
\hat{\sigma}_{1}=\left(\begin{array}{cc}
0 & g_{1}^{2} \\
g_{2}^{2} & 0
\end{array}\right), \\
\hat{\sigma}_{2}=\left(\begin{array}{cc}
0 & -i \times g_{1}^{2} \\
i \times g_{2}^{2} & 0
\end{array}\right), \\
\hat{\sigma}_{3}=\left(\begin{array}{cc}
w \times g_{1}^{2} & 0 \\
0 & w \times g_{2}^{2}
\end{array}\right) .
\end{gathered}
$$

which are known as the irregular Pauli-Santilli isomatry, and cannot any longer be constructed via non-unitary transformations of the Pauli matrices, and.

The irregular character of isomatrices (19) is established by the appearance of structure functions in the isocommutation rules 


$$
\begin{gathered}
{\left[\tilde{\sigma}_{1}, \tilde{\sigma}_{2}\right]=i \times w^{-1} \times \tilde{\sigma}_{3},\left[\tilde{\sigma}_{2}, \tilde{\sigma}_{3}\right]=i \times w \times \tilde{\sigma}_{1},} \\
{\left[\tilde{\sigma}_{3}, \tilde{\sigma}_{2}\right]=i \times w \times \tilde{\sigma}_{1},}
\end{gathered}
$$

with the characterization of the following mutation (in Santilli's words) of the SU(2) -spin eigenvalues

$$
\begin{gathered}
\tilde{\sigma}^{\hat{2}} \widehat{\times} \mid \hat{\psi}>= \\
\left(\tilde{\sigma}_{1} \times T \times \tilde{\sigma}_{1}+\tilde{\sigma}_{2} \times T \times \tilde{\sigma}_{2}+\tilde{\sigma}_{3} \times T \times \tilde{\sigma}_{3}\right) \times T \times \mid \hat{\psi}>= \\
=\left(2+w^{2}\right) \times \mid \hat{\psi}>, \\
\tilde{\sigma}_{3} \widehat{\times}\left|\hat{\psi}>=\tilde{\sigma}_{3} \times T \times\right| \hat{\psi}>= \pm w \times \mid \hat{\psi}>, w \neq 1,(21 \mathrm{~b})
\end{gathered}
$$

In essence, Santilli's irregular isorepresentation of $\hat{\mathrm{S} U}(2)$ characterize a generalization of the conventional constant values of spin 1/2 into locally variable spin isoeigenvalues.

Rather than being a mathematical curiosity, the above spin mutation is expected to be important for a consistent representation of the spin of an electron, e.g., under the immense pressures, densities and temperature in the core of a star.

\section{Independent Studies}

Numerous mathematicians have made seminal contributions to the Lie-Santilli isotheory, among whom we quote: C-X, Jiang has conducted comprehensive studies [15] on the isonumber theory at the foundation of the Lie-Santilli isotheory; D. S. Sourlas and G. T. Tsagas have conducted the first comprehensive study of the Lie-Santilli theory [16], although prior to the discovery of isonumbers [2]; J. V. Kadeisvili has studied in detail the Lie-Santilli isotheory [17] following its formulation as in memoir [3]; R. M. Fal ${ }^{\mathrm{c}^{\prime}}$ on and J. N. Valdés [18] have presented the most rigorous formulation to date of Santilli's isotopies; T. Vougiouklis [19] has developed the hyperstructural formulation of the Lie-Santilli isotheory which is the broadest possible formulation achievable with current mathematical knowledge; and S. Georgiev [20] has produced one of the most monumental works in mathematics showing the implications for all of mathematics of the isodifferential calculus which is nowadays called the Santilli-Georgiev isodifferential calculus. A comprehensive review with a large list of contributions has been produced by I. I. Gandzha and J. Kadeisvili, in monograph [21] with the suggestive title of New Sciences for a New Era.

\section{Open Problems}

The author has no words to recommend the study of regular and irregular isorepresentations of Lie-Santilli isoalgebras, with particular reference to the identification of a method for the construction of irregular isorepresentation parallel to that for the regular case of Section 4. The proposed study is important for a number of applications, such as:

\subsection{Reconstruction of Exact Symmetries}

Santilli has shown in Ref. [9] that the breaking of conventional spacetime and internal symmetries is the outcome of insufficient mathematics. because broken symmetries can be reconstructed as being exact at the covering isotopic level under the preservation of the conventional structure constants. This reconstruction has a number of important epistemological as well as technical implications. It is sufficient to note the reconstruction of parity under weak interactions or the maintaining of Einstein's abstract axioms of special relativity for interior conditions to illustrate the implications at hand. Their systematic study can be best done via the study of the isorepresentation of Lie-Santilli isoalgebras.

\subsection{Invariant Representation of Hubble's Law}

The regular Lorentz-Santilli isosymmetry has permitted an invariant derivation of the Hubble law on the cosmological redshift $\mathrm{z}=\mathrm{Hd}$ via the mere admission that light loses energy to the cold intergalactic medium without any need for the hyperbolic conjecture of the expansion of the universe via the assumption $z=H d-v / c[6,12]$. It is important to verify this occurrence via the study of the regular isorepresentations of the Lorentz-Santilli isosymmetry due to its implications for all of cosmology, since the elimination of the expansion of the universe will likely require the revision of all our cosmological knowledge.

\subsection{Synthesis of the Neutron from the Hydrogen}

In the author's view, the most important application and verification of isomathematics has been Santilli's exact and invariant representation at both the non-relativistic and relativistic levels of all characteristics of the neutron in its synthesis from the hydrogen (see review [21]). Such a synthesis is notoriously impossible for the conventional Hilbert space and related mathematics, e.g., because the rest energy of the neutron is bigger than the sum of the rest energies of the proton and the electron (a pure anathema for quantum mechanics); the Dirac equation, which is so effective for the representation of the electron orbiting around the proton in the hydrogen atom, becomes completely ineffective for the representation of the same electron when "compressed" (according to Rutherford) inside the proton; and for other reasons. The representation of the neutron synthesis was crucially dependent on the assumption of the proton and the electron as being isoparticles, that is, isounitary irreducible representations of the Galileo-Santilli or the Lorentz-Santilli isosymmetry whose study is evidently fundamental for true advances in particle physics, as well as in the structure of stars.

\subsection{Nuclear Constituents as Extended Particles}

One of the most important applications of isomathematics is the quantitative prediction of new nuclear energies without the release of harmful radiations (see review [21]). This prediction is based on the invariant representation of nuclear 
constituents as being extended and deformable charge distributions. Such a representation has been instrumental for the first achievement of the exact representation of nuclear magnetic moments and spin [7,10]. This new conception of the nuclear structure requires the representation of protons and neutrons as isoparticles. It is evident that important advances in nuclear physics and new clean energies will be curtailed until there are systematic studies on the isorepresentations of the Lie-Santilli isosymmetry.

\subsection{Elimination of the Divergencies of Quantum Mechanics}

Some of the biggest insufficiencies of quantum mechanics in particle physics are due to the singular character of Dirac's delta distribution at the origin, with ensuing divergencies of perturbative series that requiring the achievement of numerical results via the unreassuring subtraction of infinities. Santilli $[9,10]$ has shown that isotopies of Dirac's delta distribution into a function without singularities at the origin. Additionally, in all known applications the absolute value of the isotopic element (2) is very small, with the consequential capability of turning divergent or slowly convergent quantum series into rapidly convergent ones (see the infinite series of isomonomials (8) for comparison). Due to the implications of these features for all quantitative sciences, it appears recommendable that they are confirmed and further developed via the study of the isorepresentation of the Galileo-Santilli or Lorentz-Santilli isosymmetries.

\subsection{Electron Valence Bonds}

According to the axioms of quantum mechanics and chemistry, two valence electrons, rather than forming any molecular bond, should repel each other due to the Coulomb repulsion of their equal charges $\mathrm{F}=\mathrm{ke}^{2} / \mathrm{r}^{2}$ which becomes extremely strong at the distances $10^{-13} \mathrm{~cm}$ of valence bonds. Santilli [11] has achieved a strongly attractive force between two electrons in singlet valence coupling via the admission that their wavepackets is in condition of total mutual penetration, resulting in non-Hamiltonian interactions represented with isotopic elements of type (2). In view of the predictable advances for all of chemistry, it is important to verify Santilli's strong valence bond via the study of the regular isorepresentations of the Lorentz-Santilli isosymmetry characterizing the valence electrons.

\subsection{Nuclear and Chemical Reactions}

The preceding applications can be sufficiently treated via regular isorepresentations since they deal with systems of extended particles assumed as being isolated from the rest of the universe. Santilli $[9,10,11]$ has pointed out the insufficiency of the regular isorepresentations for nuclear and chemical reactions because they are irreversible over time, a feature that can only be represented via structure functions $\mathrm{w}_{i \mathrm{ith}}(\mathrm{t}, \ldots) \neq \mathrm{C}_{i \mathrm{f}}^{\mathrm{exp}}\left(\underline{\mathrm{t}}_{\mathrm{t}}, \ldots\right)$ time dependence of the type $\mathrm{C}_{\mathrm{ij}}(, \ldots) \neq \mathrm{C}_{\mathrm{ij}}(-, \ldots)$. Therefore, advances on much needed new energies without harmful radiation and on clean burning fuels will crucially depend on the availability of mathematical studies on irreducible isorepresentation of Lie-Santilli isoalgebras.

Due to their relevance, the R. M. Santilli Foundation has research funds for the writing of papers on the isorepresentations of the Lie-Santilli isotheory and their applications.

\section{Acknowledgments}

The author would like to thank Prof. R. M. Santilli for suggesting this paper, for making available the Tex files of papers in the field, and for numerous comments and suggestions.

\section{References}

[1] R. M. Santilli, Foundation of Theoretical Mechanics, Volumes I (1978) [1a], and Volume II (1982) [1b], Springer-Verlag, Heidelberg, http://www.santilli-foundation.org/docs/Santilli-209.pdf http://www.santilli-foundation.org/docs/santilli-69.pdf

[2] R. M. Santilli, "Isonumbers and Genonumbers of Dimensions 1, 2, 4, 8, their Isoduals and Pseudoduals, and "Hidden Numbers" of Dimension 3, 5, 6, 7," Algebras, Groups and Geometries Vol. 10 , 273 http://www.santilli-foundation.org/docs/Santilli-34.pdf

[3] R. M. Santilli, "Nonlocal-Integral Isotopies of Differential Calculus, Mechanics and Geometries," in Isotopies of Contemporary Mathematical Structures, P. Vetro Editor, Rendiconti Circolo Matematico Palermo, Suppl. Vol. 42, 7-82 (1996) http://www.santilli-foundation.org/docs/Santilli-37.pdf

[4] R. M. Santilli, JINR Rapid Comm. 6. 24-38 (1993), available as free download from http://www.santilli-foundation.org/docs/Santilli-19.pdf

[5] R. M. Santilli, Acta Applicandae Mathematicae 50, 177 (1998), available as free download from http://www.santilli-foundation.org/docs/Santilli-19.pdf

[6] R. M. Santilli, "Experimental Verifications of IsoRedShift with Possible Absence of Universe Expansion, Big Bang, Dark Matter, and Dark Energy," The Open Astronomy Journal 3, 124 (2010) http://www.santilli-foundation.org/docs/Santilli-isoredshift.pdf

[7] R. M. Santilli, "Nuclear realization of hadronic mechanics and the exact representation of nuclear magnetic moments," R. M. Santilli, Intern. J. of Phys. Vol. 4, 1-70 (1998) http://www.santilli-foundation.org/docs/Santilli-07.pdf

[8] R. M. Santilli, "Theoretical prediction and experimental verification of the new chemical species of magnecules," $\begin{array}{lllll}\text { Hadronic } & \text { J. } & 21, & 789 & \text { (1998) }\end{array}$ http://www.santilli-foundation.org/docs/Santilli-43.pdf

[9] R. M. Santilli, Elements of Hadronic Mechanics, Volumes I and II Ukraine Academy of Sciences, Kiev, 1995, http://www.santilli-foundation.org/docs/Santilli-300.pdf $\mathrm{http}: / / w w w . s a n t i l l i-f o u n d a t i o n . o r g / d o c s / S a n t i l l i-301 . p d f$

[10] R. M. Santilli, Hadronic Mathematics, Mechanics and Chemistry, Volumes I to V, International Academic Press, (2008), http://www.i-b-r.org/Hadronic-Mechanics.htm 
[11] R. M. Santilli, Foundations of Hadronic Chemistry, with Applications to New Clean Energies and Fuels, Kluwer Academic Publishers (2001), http: //www.santilli-foundation.org/docs/Santilli-113.pdf

[12] H. Ahmar, G. Amato, J. V. Kadeisvili, J. Manuel, G. West, and O. Zogorodnia, "Additional experimental confirmations of Santilli's IsoRedShift and the consequential expected absence of universe expansion," Journal of Computational Methods in Sciences and Engineering, 13, $321 \quad$ (2013), $\mathrm{http} / / / \mathrm{www}$.santilli-foundation.org/docs/IRS-confirmations-212 .pdf

[13] Y. Yang, J. V. Kadeisvili, and S. Marton, "Experimental Confirmations of the New Chemical Species of Santilli Magnecules," The Open Physical Chemistry Journal Vol. 5, $1-16$

http://www.santilli-foundation.org/docs/Magnecules-2012.pdf for the industrial realization of Santilli magnecules, please visit the website of the publicly traded U. S. company Magnegas Corporation www.magnegas.com

[14] S. S. Dhondge, "Studies on Santilli Three-Body Model of the Deuteron According to Hadronic Mechanics," American Journal of Modern Physics, in press (2015) http://www.santilli-foundation.org/docs/deduteron-cpnfirm.pd $\mathrm{f}$ For the industrial realization of nuclear energies without harmful radiation, please visit the website of the publicly traded U. S. company Thunder Energies Corporation www.thunder-energies.comn
[15] Chun-Xuan Jiang, Foundations of Santilli Isonumber Theory, International Academic Press (2001), http://www.i-b-r.org/docs/jiang.pdf

[16] D. S. Sourlas and G. T. Tsagas, Mathematical Foundation of the Lie-Santilli Theory, Ukraine Academy of Sciences (1993) http://www.santilli-foundation.org/docs/santilli-70.pdf

[17] J. V. Kadeisvili, "Foundations of the Lie-Santilli Isotheory," in "Isotopies of Contemporary Mathematical Structures," P. Vetro Editor, Rendiconti Circolo Matematico Palermo, Suppl. Vol. 42, 7-82 http://www.santilli-foundation.org/docs/Santilli-37.pdf

(1996),

[18] R. M. Fal ${ }^{\mathrm{c}^{\prime}}$ on and J. N. Valdés, Mathematical Foundations of Santilli Isotopies, Original monograph in Spanish published in 2001. The English translation has been published inAlgebras, Groups and Geometries Vol. 32, pages 135-308 (2015) http://www.i-b-r.org/docs/Aversa-translation.pdf

[19] T. Vougiouklis, "The Santilli theory 'invasion' in hyperstructures," Algebras, Groups and Geometries Vol. 28, pages 83-104 (2011) http://www.santilli-foundation.org/docs/santilli-invasion.pdf

[20] S. Georgiev, Foundations of the IsoDifferential Calculus, Volumes I to V, Nova Scientific Publishers (2014 on ).

[21] I. Gandzha and J. Kadeisvili, New Sciences for a New Era: Mathematical, Physical and Chemical Discoveries of Ruggero Maria Santilli, Sankata Printing Press, Nepal (2011), http://www.santilli-foundation.org/docs/RMS.pdf 